\title{
Collagen Diseases (C. D.) Analysed from the Standpoint of Subacute Bacterial Endocarditis (SBE)
}

\author{
Masataka KATsU \\ Department of Internal Medicine (Director: Prof. Ittaku Mikata), \\ School of Medicine, Keio University
}

\section{Introduction}

In 1944, Albertini had stated that series of different diseases could be induced as the results of equilibrium between virulence of bacteria and resistance of host on the basis of rabbit experiment using hemolytic streptococci. From this experiment, he had plotted series of different diseases on his well-cited semi-circled schematic diagram. This schematic diagram was modified by several authors such as Mach (1951), Heilmeyer (1954) and Fanconi (1959) (Fig. 1).

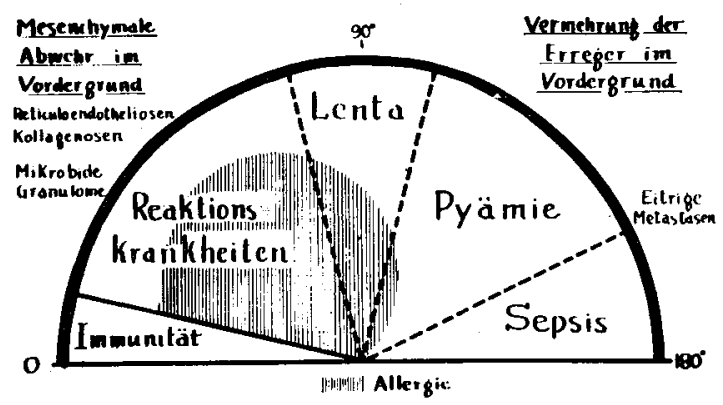

Fig. 1. Semi-circled schematic diagram by G. Fanconi (1959).

These diagrams illustrate that SBE stands very close to collagen diseases in the sense of so called allergic reaction to be called disease of reaction and/or mesenchymal reaction.

In our clinical practice, indeed, we encounter not infrequently the cases in which it is difficult to differentiate chronic septicemia such as SBE or miliary tuberculosis from collagen diseases and vice versa. Purpose of this paper is to clarify the similarity and difference of the two from various angles. 


\section{Pathological studies-Experimental bacterial endocarditis (EBE) and autopsy cases-}

Experiments were performed in order to induce bacterial endocarditis in rabbits which were divided into three groups such as the one treated with bacteria (Strep. viridans) alone, sensitizing agents only (horse sera and nephrotoxin) and both. The higher incidences of endocarditis and fibrinoid degeneration were noted among the group of animals which were treated by the combination of bacteria and sensitizing agent, suggesting synergestic action of the two.

This fact might indicate that SBE is not only disease caused by bacterial invasion but also disease modified by host-reaction. Histologic examination was performed in 26 cases of SBE, 10 of miliary tuberculosis, 10 of acute septicemia, 9 of systemic lupus erythmatosus (SLE), 6 of periarteritis nodosa (PN), 2 of scleroderma, 7 of rheumatic fever (RF) and 1 of rheumatoid arthritis (RA), amounting 71 cases. In SLE and PN, the histologic changes in heart, lung, spleen, and especially in kidney, were more marked than those in septic diseases. Reviewing each case separately, however, histologic changes akin to those seen in collagen diseases were also noted not infrequently even among SBE and/or septicemia.

\section{Clinical studies}

A total of 54 cases (16 of SLE, 5 of PN, 3 of SD, 26 of SBE, one of acute septicemia, and 3 of miliary tuberculosis) were studied. Signs and symptoms most commonly seen of all cases were tabulated (Table 1). In general, there were many cases which were rather easily diagnosed by having specific signs and symptoms and laboratory findings such as positive blood culture or LE cell phenomenon and appearance of typical butterfly shaped erythema etc. among both groups of diseases. But non-specific or rather overlapped ones are occasionally seen, making differential diagnosis difficult before autopsy. Cases studied were as follows: Case 1 was thought to have unclassified collagen disease but autopsy revealed to be miliary tuberculosis. Clinical diagnosis of case 2 was 
thrombocytopenic purpura or $\mathrm{PN}$, but pathologically it was SIE with thrombotic thrombocytopenic purpura. A case which was thought to have SBE clinically was the third one, but autopsy specimen showed it to be SLE.

\section{Laboratory studies}

a) Latex Fixation Test (LFT) and Bentonite Flocculation Test (BFT)

LFT and BFT were performed on the case of SBE and C.D., revealing that these tests reacted positively in about $50 \%$ of the cases in the both conditions. But of note, in 51 cases of other infectious diseases including acute septicemia, none reacted positively.

b) Serum iron (Se-Fe) and serum copper ( $\mathrm{Se}-\mathrm{Cu}$ )

In acute febrile stage of SBE and septicemia, decrease of Se-Fe and increase of $\mathrm{Se}-\mathrm{Cu}$ were rather marked making mirror image pattern, but in collagen diseases excluding $R A$ and $R F$, increase of $\mathrm{Se}-\mathrm{Cu}$ was not seen although Se-Fe showed same attitude to the former.

c) Column chromatography and paper electrophoresis (Fig. 2)

Six cases of RA, 3 of SLE, 2 of PN, 1 of SD, 7 of SBE, and 2 of healthy adult totalling 21 cases were subjected to both tests. In column chromatography, three major peaks were eluted by different buffer solutions. Peak 1 was eluted at PH 8.0-7.0 and was composed mainly of gamma-globulin electrophoretically. Ultracentrifugal studies were performed by the coutesy of National Institute of Animal Health and peak 1 was revealed to be $6.2 \mathrm{~S}$ globulin. Peak 2 eluted at pH 5.0 was composed mainly of albumin. Peak 3 was eluted at pH 4.6 and was gamma-globulin electrophoretically. In ultracentrifugation, it was $17.2 \mathrm{~S}$ with the specimen obtained from patient with definite RA.

Euglobulin fraction from healthy adult was also tested. In chromatographic and electrophoretic patterns, it lacked albumin portion (2nd peak). But it was of note that globulin existed widely spread out in whole area although it was small amount.

BFT was performed by the use of each fraction. In all case of RA, bentonite flocculating substance was detected only at peak 3 . 


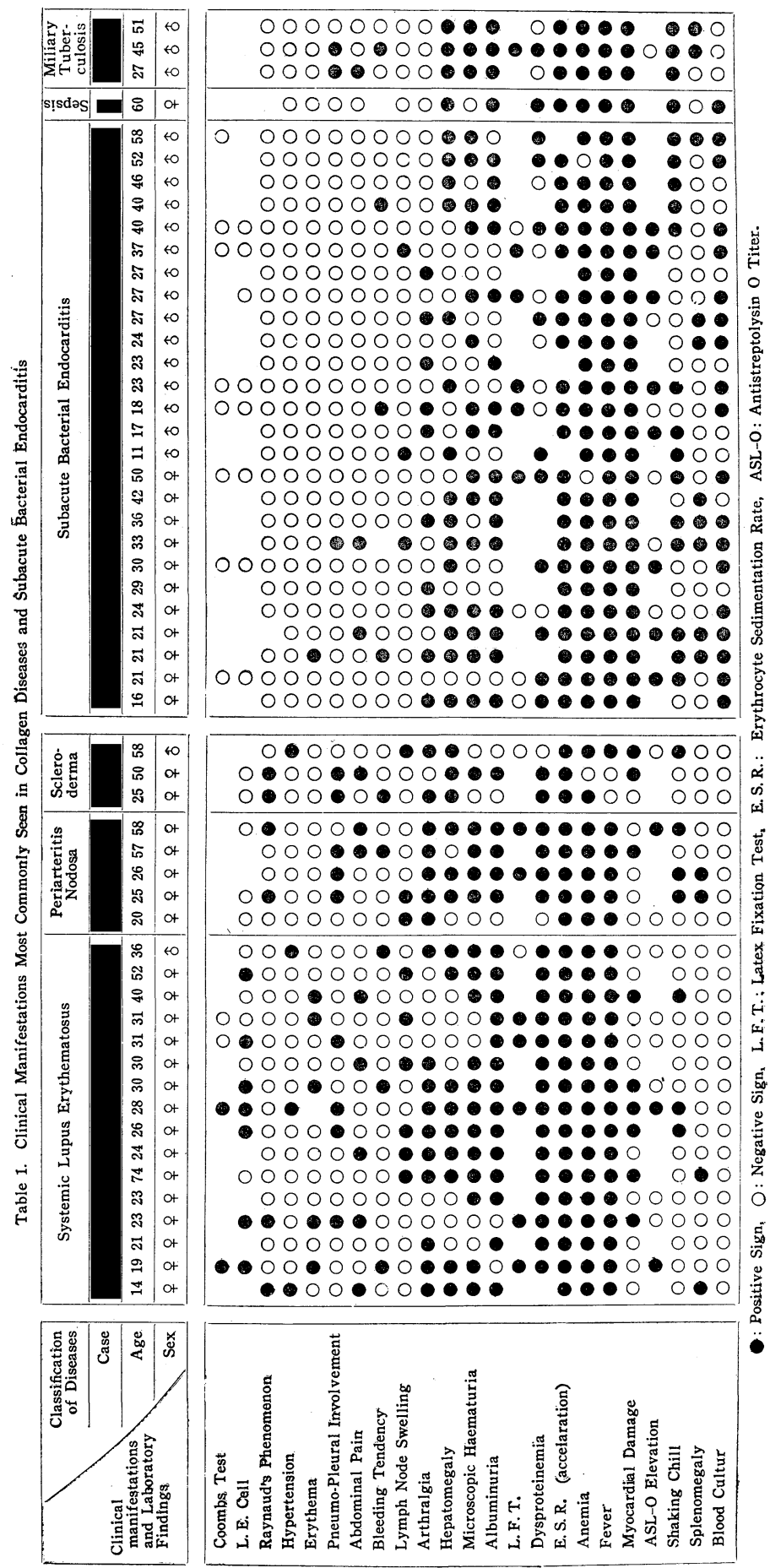


In $\mathrm{SBE}$, this substance was detected in every fraction eluted at all $\mathrm{pH}$ ranges ( $\mathrm{pH} 8.0-4.6)$.

Peak 1 from SBE which showed flocculating activity was subjected to ultracentrifugal study. It was composed of $6.3 \mathrm{~S}$ and $16.6 \mathrm{~S}$. Bentonite flocculating substance in collagen diseases such as SLE and $\mathrm{PN}$, on the contrary, appeared in few places mostly two. Different attitude of this substance to sensitized bentonite in SBE in comparison with SLE and PN was the appearance of this substance in the fractions eluted even with $\mathrm{pH} 8.0-7.0$ (Fig. 2).

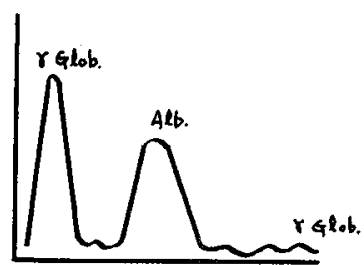

Normal

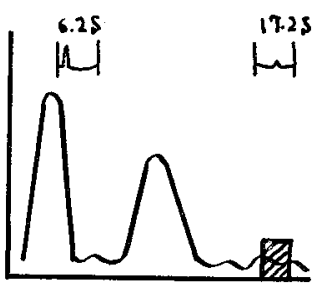

$\mathrm{RA}$ (mean pattern of 6 cases)

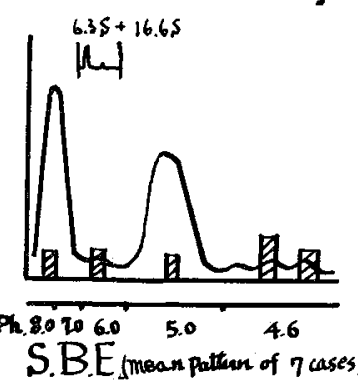

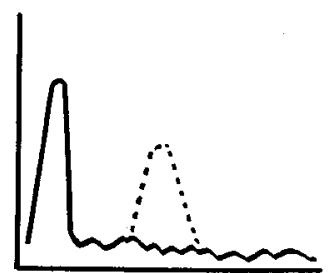

Euglobulin

Fig. 2. Distribution of bentonite flocculating substances.

\section{Conclusion}

Since SBE is considered to be caused by bacteria of low-grade virulence, clinical and laboratory manifestations are strongly modified by vital reaction of the host. Such being the case, it is of no doubt that SBE has clinical and laboratory similarities to collagen diseases as the disease of reaction.

On the other hand, definite differences exist between SBE and collagen diseases. Firstly, there is an etiological difference. Septicemia and/or SBE are diseases directly related to bacterial invasion. Secondly, there is a therapeutic difference-that is, in sep- 
ticemia and/or SBE antibiotics plays the major role and single use of adrenocortical steroid hormones (glucocorticoids), which is effective to collagen diseases, is principally contra-indicated, although in some cases beneficial influence can be obtained from the combined use of antibiotics and steroid hormones. Clinicians, as we are, not only try to seek similarity of these two diseased conditions but also try to find the way of how to differentiate the two and give proper treatment in earlier stage. 